\title{
A cost-utility analysis of transcatheter versus surgical aortic valve replacement for the treatment of aortic stenosis in the population with intermediate surgical risk
}

Derrick Y. Tam, MD, ${ }^{\text {a,b }}$ Avery Hughes, BSocSci, ${ }^{\text {b,c }}$ Stephen E. Fremes, MD, MSc, ${ }^{\text {a,b }}$ Saerom Youn, HBSc, ${ }^{b}$ Rebecca L. Hancock-Howard, PhD, ${ }^{\mathrm{b}}$ Peter C. Coyte, $\mathrm{PhD},{ }^{\mathrm{b}}$ and Harindra C. Wijeysundera, MD, PhD ${ }^{\mathrm{b}, \mathrm{c}, \mathrm{d}}$

\section{ABSTRACT}

Objective: Although transcatheter aortic valve implantation has been shown to be noninferior to surgical aortic valve replacement in patients with severe aortic stenosis at intermediate surgical risk, the cost-effectiveness of this strategy in this population is unknown. Our objective was to conduct a cost-utility analysis comparing transcatheter aortic valve implantation with surgical aortic valve replacement in the population with intermediate risk severe aortic stenosis.

Methods: A fully probabilistic Markov model with 30-day cycles was constructed from the Canadian third-party payer's perspective to estimate the difference in cost and effectiveness (measured as quality-adjusted life years) of transcatheter aortic valve implantation versus surgical aortic valve replacement for intermediate-risk patients over a lifetime time horizon, discounted at $1.5 \%$ per annum. Clinical trial data from The Placement of Aortic Transcatheter Valve 2 informed the efficacy inputs. Costs (adjusted to 2016 Canadian dollars) were obtained from the Canadian Institute of Health Information and the Ontario Schedule of Benefits. Incremental cost-effectiveness ratios were calculated.

Results: In the base-case analysis, total lifetime costs for transcatheter aortic valve implantation were $\$ 10,548$ higher than surgical aortic valve replacement but added 0.23 quality-adjusted life years, for an incremental cost-effectiveness ratio of $\$ 46,083 /$ quality-adjusted life-years gained. Deterministic 1-way analyses showed that the incremental cost-effectiveness ratio was sensitive to rates of complications and cost of the transcatheter aortic valve implantation prosthesis. There was moderate-to-high parameter uncertainty; transcatheter aortic valve implantation was the preferred option in only $52.7 \%$ and $55.4 \%$ of the simulations at a $\$ 50,000$ and $\$ 100,000$ per quality-adjusted life years willingness-to-pay thresholds, respectively.

Conclusions: On the basis of current evidence, transcatheter aortic valve implantation may be cost-effective for the treatment of severe aortic stenosis in patients with intermediate surgical risk. There remains moderate-to-high uncertainty surrounding the base-case incremental cost-effectiveness ratio. $(\mathrm{J}$ Thorac Cardiovasc Surg 2018;155:1978-88)

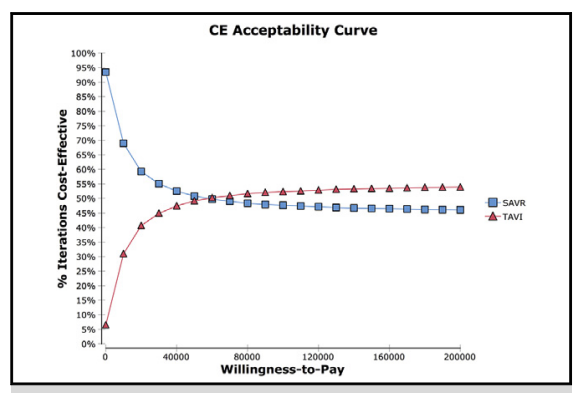

Cost-effectiveness acceptability curve for TAVI versus SAVR.

\section{Central Message}

TAVI may be cost-effective in the population with intermediate surgical risk compared with SAVR.

\section{Perspective}

Clinical trials in patients with severe AS at intermediate surgical risk showed noninferiority when TAVI was compared with SAVR. However, the cost-effectiveness of TAVI in this population was not known. This analysis showed that TAVI may be an economically attractive option compared with SAVR in this population.

See Editorial Commentary page 1989.

See Editorial page 1977.

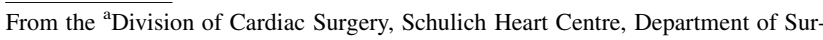
gery, Sunnybrook Health Sciences Centre, University of Toronto, Toronto, Ontario, Canada; ' Institute of Health Policy, Management and Evaluation, University of Toronto, Toronto, Ontario, Canada; ${ }^{\mathrm{c}}$ Program of Child Health Evaluative Services, The Hospital for Sick Children, Peter Gilgan Centre For Research and Learning, University of Toronto, Toronto, Ontario, Canada; and ${ }^{\mathrm{d}}$ Division of Cardiology, Schulich Heart Centre, Department of Medicine, Sunnybrook Health Sciences Centre, University of Toronto, Toronto, Ontario, Canada.

D.Y.T. is supported by the Ontario Ministry of Health Clinician Investigator's Program (Toronto, Ontario). A.H. is supported by the SickKids-University of Toronto Ontario Student Opportunity Trust Fund (RestraComp) Award (Toronto, Ontario). S.E.F. is supported by the Bernard S. Goldman Chair in Cardiovascular Surgery
(Toronto, Ontario). H.C.W. is supported by a Distinguished Clinician Scientist Award for the Heart and Stroke Foundation of Canada (Ottawa, Canada). Read at the 43rd Annual Meeting of The Western Thoracic Surgical Association, Colorado Springs, Colorado, June 21-24, 2017.

Received for publication May 11, 2017; revisions received Oct 31, 2017; accepted for publication Nov 24, 2017; available ahead of print Feb 14, 2018.

Address for reprints: Harindra C. Wijeysundera, MD, PhD, Schulich Heart Centre, Sunnybrook Health Sciences Centre, 2075 Bayview Ave, Room A202, Toronto, Ontario M4N 3M5, Canada (E-mail: harindra.wijeysundera@sunnybrook.ca). $0022-5223 / \$ 36.00$

Copyright (C) 2018 by The American Association for Thoracic Surgery https://doi.org/10.1016/j.jtcvs.2017.11.112 


\section{Abbreviations and Acronyms \\ AS $=$ aortic stenosis \\ FDA $\quad=$ Food and Drug Administration \\ GDP $=$ gross domestic product \\ ICER = incremental cost-effectiveness ratio \\ ICU = intensive care unit \\ PARTNER $=$ The Placement of Aortic Transcatheter Valve \\ QALY = quality-adjusted life year \\ QoL $=$ quality of life \\ RCT $=$ randomized clinical trial \\ SAVR = surgical aortic valve replacement \\ STS = Society of Thoracic Surgeons \\ TAVI $=$ transcatheter aortic valve implantation \\ WTP = willingness-to-pay}

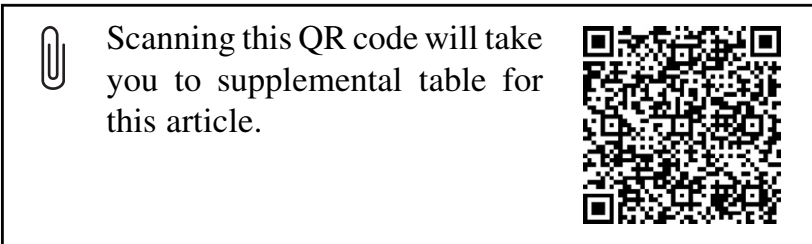

Traditionally, surgical aortic valve replacement (SAVR) was considered the gold standard for the treatment of severe aortic stenosis (AS). However, transcatheter aortic valve implantation (TAVI) has emerged as the treatment of choice for patients with severe symptomatic AS who are inoperable or at high surgical risk. ${ }^{1,2}$ Numerous previously published economic studies suggest that TAVI is costeffective in both the inoperable and high-risk populations. ${ }^{3}$ The exponential growth of TAVI has led to indication expansion to low-risk patients. A landmark randomized clinical trial (RCT) conducted in patients with intermediate surgical risk (Society of Thoracic Surgeons [STS] Predicted Risk of Mortality at 30 days 4\%-8\%) showed noninferiority for TAVI compared with SAVR. ${ }^{4}$ These findings have been reinforced by a number of propensity-matched studies and meta-analyses, ${ }^{5}$ all of which have led to regulatory approval in the United States by the Food and Drug Administration (FDA) for this expanded indication.

The intermediate-risk patients differ from the higher-risk population in several ways that may affect costs and outcomes. In particular, given that this population may be younger with fewer comorbidities, complication rates and length of hospital stay are likely to be different. Although the intermediate-risk population represents an emerging indication for TAVI, the cost-effectiveness of TAVI compared with SAVR is not known in this patient population. Accordingly, to address this gap in knowledge, we conducted a formal cost-utility analysis incorporating both lifetime costs and benefits to determine the costeffectiveness of TAVI compared with SAVR in the intermediate-risk population from the Canadian thirdparty payer perspective.

\section{MATERIALS AND METHODS \\ Model Overview}

A fully probabilistic Markov model with cycle lengths of 30 days was constructed from the Canadian third-party payer's perspective to estimate the cost and effectiveness of TAVI versus SAVR for the intermediate-risk patient population over a lifetime time horizon. Under Canada's universal health care plan, the provinces are responsible for all care delivery; thus, in Ontario, the third-party payer is the Ministry of Health and Long Term Care. The primary cost outcome was total lifetime cost measured in 2016 Canadian Dollars and the primary effectiveness outcome was quality-adjusted life-years (QALYs). QALYs were obtained by multiplying the time spent in a particular health state with the quality weight for that respective health state. The incremental cost-effectiveness ratio (ICER) was calculated by taking the differences in lifetime costs between the 2 treatment groups (ie, incremental costs) and dividing by the differences in lifetime benefits (ie, incremental effectiveness, measured in QALYs). All outcomes and costs were discounted at $1.5 \%$ per annum in accordance with the Canadian Agency for Drugs and Technologies in Health guidelines. $^{6}$

\section{Model Structure}

All patients entered the model in the procedural state (TAVI or SAVR) and were at risk for clinically relevant short-term complications (major bleeding, acute kidney injury, vascular injury, atrial fibrillation, nondisabling stroke, rehospitalization) and long-term complications (death, dialysis, and disabling stroke).

After the procedural state, all patients transitioned into 1 of 4 long-term states (alive and well, disabling stroke, dialysis, or death) as shown in Figure 1. Repeat hospitalization was a transition for which a patient would remain for a proportion of 1-cycle. Patients transitioned between these long-term states on the basis of efficacy data from the results of the recently published The Placement of Aortic Transcatheter Valve (PARTNER) 2 Trial. Transition probabilities between the alive and well state and the various complication states (excluding death) occurred only in the first 2 years of the model. After 2 years, we made the conservative assumption

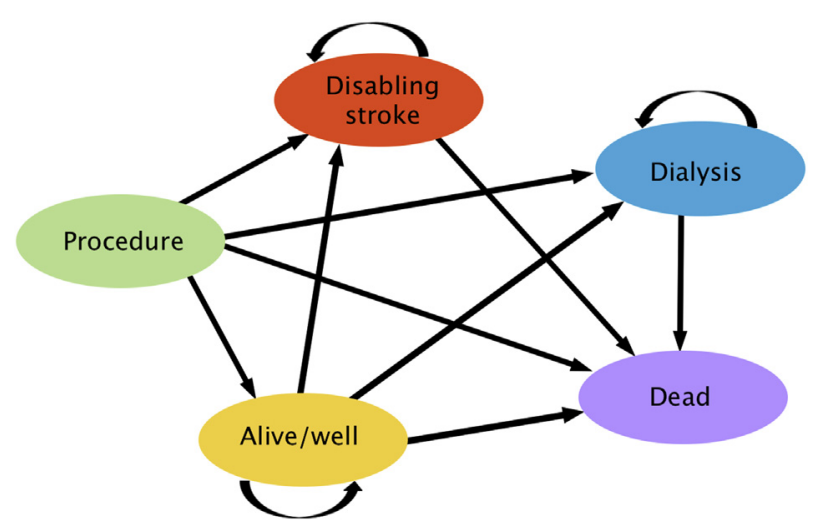

FIGURE 1. A state diagram of the Markov model. All patients started the model in the procedural state and transitioned to one of the long-term health states after 30 days postprocedure. With the exception of the dead state, patients transitioned between the different states every 30 days according to efficacy data obtained from the PARTNER 2 Trial. 
that the rates of complications were assumed to be equivalent in both the TAVI and SAVR arms. In addition, after 2 years, mortality rates in the model were based on 2010-2012 age- and gender- specific Canadian life tables given the absence of clinical trial data from the PARTNER 2 study beyond the 2-year follow-up. ${ }^{7}$ When transition probabilities were not available from PARTNER 2, published results from the literature for similar patient populations were used as described next.

\section{Clinical Efficacy Inputs}

Trial overview. The PARTNER 2 Trial was a multicenter RCT of 57 centers in the United States and Canada comparing TAVI with SAVR in the intermediate surgical risk population (defined as STS Predicted Risk of Mortality 4 of $8 \%$ at 30 days). A total of 2032 patients at intermediate surgical risk were randomized to TAVI $(\mathrm{n}=1011)$ or SAVR $(\mathrm{n}=1021)$ and followed for the primary outcome of all-cause death or disabling stroke at 2 years using a noninferiority design. The average STS risk scores in both groups were similar (TAVI 5.8 \pm 2.1 , SAVR $5.8 \pm 1.9$ ), and the average age of the cohort was $81.5 \pm 6.7$ years and $81.7 \pm 6.7$ years in the TAVI and SAVR groups, respectively. In the TAVI group, patients underwent transfemoral or transthoracic placement of the balloon-expandable Sapien XT valve system (Edwards Lifesciences, Irvine, Calif).

Efficacy end points. The 30-day clinical end points were obtained from the PARTNER 2 Trial intention-to-treat cohort: death from any cause, any stroke, disabling stroke, nondisabling stroke, rehospitalization, major vascular complication, life-threatening or disabling bleed, acute kidney injury, and new-onset atrial fibrillation (Table E1). After 30 days, transition probabilities were based on 1-year and 2-year end points from the trial. We excluded patients who had an event at 30 days and 1 year from both the numerator and denominator of cumulative long-term probabilities (1 year and 2 years, respectively) to avoid double counting patient events as described in a previous cost-analysis. ${ }^{8}$ The proportion of patients with acute kidney injury progressing to dialysis was not provided in the
PARTNER 2 Trial and was estimated from the PARTNER 1A trial; we used the same proportion in both groups. ${ }^{2}$ The probability of death during long-term dialysis was estimated from the published literature, ${ }^{9}$ and the probability of death in patients with long-term strokes was also obtained from the literature. ${ }^{10}$

Cost inputs. Up-front procedural costs included the following: TAVI system, SAVR valve, interventional cardiology procedural fees, surgeon fee, surgical assistant fee, anesthesiologist fee, ward, and intensive care unit (ICU) stay (Table 1). On the basis of input from TAVI implanters, the corresponding billing codes from the Ontario Schedule of Benefits used for TAVI and SAVR were determined. ${ }^{11}$ Ward stay and ICU stay costs were obtained from St Michael's Hospital (Toronto, Ontario, Canada) (Cardiovascular ICU Unit Clerk, St Michael's Hospital, personal communication, November 2015). Length of stay in the ICU and ward was obtained from PARTNER $2 .{ }^{4}$ Costs for both the TAVI valve system and the surgical valve were obtained from the device manufacturer, Edwards Lifesciences (Edwards Life Sciences Market Access Team, personal communication, November 2016). The costs for periprocedural complications were obtained from the 2014 Canadian Institute for Health Information Patient Cost Estimator Case Mix Group for those aged more than 80 years in Ontario (Table 2). ${ }^{12,13}$ Costs for long-term complication states of disabling stroke and dialysis were estimated from the literature, ${ }^{14,15}$ whereas hospitalization was estimated from Canadian Institute for Health Information Patient Cost Estimator 2014. All costs were inflated to 2016 Canadian dollars using the Bank of Canada Consumer Price Index $(\$ 1.00$ Canadian $=\$ 0.76$ US Dollar [Bank of Canada Currency Exchange Rate, January 7, 2017]).

Utilities and quality of life inputs. Quality of life (QoL) utilities for the different health states were obtained from the literature given the absence of specific QoL data from the PARTNER 2 trial (Table 2). ${ }^{16-21}$ We used published EuroQol data from the PARTNER 1A trial ${ }^{21}$ as a surrogate for baseline, 6-month, and 1-year utilities in the alive/well state in our

TABLE 1. Procedure-related costs and resources

\begin{tabular}{|c|c|c|c|c|c|c|}
\hline \multirow{2}{*}{$\begin{array}{l}\text { Procedure } \\
\text { Resource }\end{array}$} & \multicolumn{3}{|c|}{ TAVI } & \multicolumn{3}{|c|}{ SAVR } \\
\hline & Cost (SD) & $\begin{array}{l}\text { Mean units } \\
\text { (SD) }\end{array}$ & Source & Cost (SD) & Mean units (SD) & Source \\
\hline Valve & $\$ 24,000.00$ & 1.004 & $\begin{array}{l}\text { Edwards Lifesciences } \\
\quad \text { (Irvine, Calif) }\end{array}$ & $\$ 6000$ & 1 & Edwards Lifesciences \\
\hline Ward stay & $\$ 945(203) / d$ & $4(1.33) \mathrm{d}$ & St Michael's Hospital & $\$ 945(203) / d$ & $5(1.67) \mathrm{d}$ & St Michael's Hospital \\
\hline ICU stay & $\$ 3161(1283) / d$ & $2(0.67) \mathrm{d}$ & St Michael's Hospital & $\$ 3161(1283) / d$ & $4(1.33) \mathrm{d}$ & St Michael's Hospital \\
\hline $\begin{array}{l}\text { Aortic valve replacement - } \\
\text { Surgeon fee }\end{array}$ & $\$ 1037$ & 1 & OHIP R738 & $\$ 1403$ & 1 & OHIP R738, E650 \\
\hline First assist fee* & \$12/unit & 42 units & OHIP R738 & \$12.04/unit & 66 units & OHIP R738 \\
\hline Second assist fee* & \$12/unit & 42 units & OHIP R738 & \$12.04/unit & 66 units & OHIP R738 \\
\hline $\begin{array}{l}\text { Aortic valve replacement - } \\
\text { Anesthesia* }\end{array}$ & \$15/unit & 60 units & OHIP R738 & \$15.01/unit & 84 units & OHIP R738 \\
\hline $\begin{array}{l}\text { Insertion of temporary } \\
\text { pacemaker wire }\end{array}$ & $\$ 154$ & 0.5 & OHIP Z443 & & & \\
\hline Angiogram & $\$ 119$ & 1 & OHIP G297 & & & \\
\hline $\begin{array}{l}\text { Percutaneous angioplasty of } \\
\text { the aortic valve }\end{array}$ & $\$ 488$ & 1 & OHIP Z448A & & & \\
\hline $\begin{array}{l}\text { Retrograde aortic left heart } \\
\text { catheterization }\end{array}$ & $\$ 210$ & 0.5 & OHIP Z440 & & & \\
\hline Total procedural cost & $\$ 40,274$ & & & $\$ 29,856$ & & \\
\hline
\end{tabular}

TAVI, Transcatheter aortic valve implantation; $S A V R$, surgical aortic valve replacement; $S D$, standard deviation; $I C U$, intensive care unit; $O H I P$, Ontario Health Insurance Benefit Schedule of Benefit. *Payment for assistants and anesthesiology is based on initial baseline units for a procedure and the amount of time spent performing the procedure (estimated total procedural time as $3 \pm 1$ hours for TAVI and $5 \pm 1.67$ hours for SAVR). 
TABLE 2. Cost and utilities for short-term transitions and long-term health states

\begin{tabular}{|c|c|c|c|c|}
\hline State/transition & Cost (SD) & Source & Utility (SD) & Source \\
\hline Alive/well - SAVR & 0 & NA & $\begin{array}{l}\text { <7 mo: } 0.68(0.24) \\
7-12 \text { mo: } 0.75(0.27) \\
>12 \text { mo: } 0.74(0.23)\end{array}$ & Reynolds and colleagues $^{21}$ \\
\hline Alive/well - TAVI & 0 & NA & $\begin{array}{l}<7 \text { mo: } 0.74(0.24) \\
7-12 \text { mo: } 0.76(0.20) \\
>12 \text { mo: } 0.75(0.22)\end{array}$ & Reynolds and colleagues ${ }^{21}$ \\
\hline \multicolumn{5}{|c|}{ Long-term costs and utilities (per cycle) } \\
\hline $\begin{array}{l}\text { Hospitalization } \\
\text { Dialysis } \\
\text { Disabling stroke }\end{array}$ & $\begin{array}{r}\$ 25,255(8418) \\
\$ 15,898(5299) \\
\$ 3260(1087)\end{array}$ & $\begin{array}{c}\text { CIHI } 2014 \text { PCE } \\
\text { Wong and colleagues }(2014)^{14} \\
\text { Ohinmaa and colleagues } \\
(2016)^{15}\end{array}$ & $\begin{array}{l}0.56(0.23) \\
0.44(0.32) \\
0.39(0.12)\end{array}$ & $\begin{array}{l}\text { Ambrosy and colleagues } \\
\text { Lee and colleagues } \\
{ }^{19} \\
\text { Tengs and } \operatorname{Lin}^{18}\end{array}$ \\
\hline \multicolumn{5}{|c|}{ Transition costs and rewards } \\
\hline Disabling stroke & $\$ 9958(3319)^{*}$ & CIHI 2014 PCE & $\mathrm{NA} \dagger$ & NA \\
\hline Hospitalization & $\$ 25,255(8418)$ & CIHI 2014 PCE & $-0.1276(0.0259)$ & Lanitis and colleagues ${ }^{17}$ \\
\hline Major bleeding & $\$ 2518(839)$ & CIHI 2014 PCE & $-0.447(0.149)$ & Kaier and colleagues ${ }^{16}$ \\
\hline Vascular injury & $\$ 10,500(3500)$ & CIHI 2014 PCE & $-0.0463(0.0154)$ & Kaier and colleagues ${ }^{16}$ \\
\hline Acute kidney injury & $\$ 7450(2483)$ & CIHI 2014 PCE & $-0.177(0.059)$ & Kaier and colleagues ${ }^{16}$ \\
\hline Atrial fibrillation & $\$ 4704(1568)$ & CIHI 2014 PCE & $-0.0377(0.01257)$ & Kaier and colleagues ${ }^{16}$ \\
\hline Nondisabling stroke & $\$ 3815(1272)$ & CIHI 2014 PCE & $-0.161(0.0537)$ & Kaier and colleagues ${ }^{16}$ \\
\hline
\end{tabular}

All values are reported in Canadian dollars, 2016. When unavailable, standard deviations were derived as one third of the mean value per Saw and colleagues. ${ }^{22} S D$, Standard deviation; SAVR, surgical aortic valve replacement; NA, not applicable; TAVI, transcatheter aortic valve implantation; $C I H I$, Canadian Institute for Health Information; PCE, Patient Cost Estimator. *Average of "average of hemorrhagic event" and "ischemic event of CNS" in Ontario. †Transition into disabling stroke was assigned only transition costs because it is a long-term state and disutility is captured in utility assigned to disabling stroke state.

model. Utilities for long-term Markov states were estimated from the literature for hospitalization, ${ }^{20}$ dialysis, ${ }^{19}$ and disabling stroke. ${ }^{18}$

For periprocedural complications, we assigned a 1-time decrement in utility (ie, disutility), which represented a decreased QoL for that particular complication. These disutilities were estimated from a prospective observational cohort study of TAVI and SAVR cases published in the literature for major bleeding, vascular complications, acute kidney injury, atrial fibrillation, and nondisabling stroke. ${ }^{16}$ The disutility for hospitalization was estimated from a French cost-effectiveness model of patients with atrial fibrillation. ${ }^{17}$

Base-case and sensitivity analyses. The base case was conducted fully probabilistically, with 10,000 Monte-Carlo simulations. All inputs described were sampled from distributions, with the expected value being the mean from the studies and the standard deviations from the source literature. For any data without standard deviations, we used one third of the point estimate as the standard deviation, as previously described. ${ }^{22}$ For all probabilities and utilities, a beta distribution was used, whereas a gamma distribution was used for costs, procedural length, and hospital length of stay. The average costs and effects for the 10,000 simulations were used to calculate the base-case results and ICER. Parameter uncertainty was represented by a cost-effectiveness acceptability curve, showing the proportion of the simulations that favored TAVI at different willingness-to-pay (WTP) thresholds.

A series of deterministic 1-way sensitivity analyses were also performed to examine the impact of key parameters in the model on the ICER. For probabilities, $95 \%$ confidence intervals were derived, and the lower and upper boundaries were used as the low and high values in the deterministic 1 -way SA. For costs, the lower and upper values were $\pm 50 \%$ of the estimated costs. In addition to the base-case analysis ICER, we performed a scenario analysis restricted to the transfemoral-access only cohort compared with the SAVR cohort ( $\mathrm{n}=775$ in both groups). Efficacy inputs were informed from the periprocedural, 1-year, and 2-year event rates from the transfemoral access and SAVR group from the PARTNER 2 Trial. ${ }^{4}$

\section{RESULTS}

In the base-case analysis, the index hospitalization cost in the TAVI group was $\$ 40,274$ compared with $\$ 29,856$ in the SAVR group. With $1.5 \%$ global discounting, the total lifetime cost was $\$ 46,904 \pm \$ 4038$ and $\$ 36,356 \pm \$ 7309$ in the TAVI and SAVR groups, respectively. The life-years gained were $7.57 \pm 0.10$ and $7.42 \pm 0.11$ for TAVI and SAVR, respectively. When QoL was taken into account, the QALYs gained were $5.63 \pm 1.47$ and $5.40 \pm 1.47$ for TAVI and SAVR, respectively (Table 3 ). Thus, the incremental cost was $\$ 10,547$ and the incremental gain in QALY was 0.23. This resulted in an ICER of $\$ 46,083 /$ QALY after discounting.

The total undiscounted lifetime costs were $\$ 47,054 \pm \$ 4113$ and $\$ 36,478 \pm 7261$ for TAVI and SAVR, respectively, for a difference in total costs of $\$ 10,576$. The total undiscounted QALYs gained for TAVI and SAVR were $6.18 \pm 1.60$ and $5.92 \pm 1.63$, respectively, resulting in a difference of 0.27 QALYs. Thus, the ICER without discounting was $\$ 39,661 /$ QALY.

In 1-way sensitivity analyses, costs and procedural complication rate thresholds were calculated for a WTP of $\$ 50,000 /$ QALY gained. The ICER exceeded \$50,000/ QALY gained when the cost of the TAVI valve system was greater than $\$ 25,100$ (compared with base case of $\$ 24,000$ ) or when TAVI ICU length of stay was greater than 2.3 days (base case of 2 days). For procedural complications, the ICER was greater than $\$ 50,000 /$ QALY when 
TABLE 3. Results of base-case analysis

\begin{tabular}{lrcr}
\hline \multicolumn{1}{l}{ Treatment arm } & Total cost & Total effectiveness (QALYs) & $\begin{array}{c}\text { Incremental cost/incremental } \\
\text { effectiveness }\end{array}$ \\
\hline $\begin{array}{l}\text { Discounted costs and QALYs } \\
\text { TAVI }\end{array}$ & & \\
SAVR & $\$ 46,904 \pm 4038$ & $5.63 \pm 1.47$ & $\$ 10,547 / 0.23$ \\
Undiscounted costs and QALYs & $5.40 \pm 1.47$ & \\
TAVI & $\$ 47,054 \pm 4113$ & & \\
SAVR & $\$ 36,478 \pm 7261$ & $6.18 \pm 1.60$ & $\$ 10,576 / 0.27$ \\
\hline
\end{tabular}

QALY, Quality-adjusted life-years; ICER, incremental cost-effectiveness ratio; TAVI, transcatheter aortic valve implantation; SAVR, surgical aortic valve replacement.

the rate of periprocedural mortality in the TAVI was greater than $4.2 \%$ or less than $3.6 \%$ in the SAVR arm and the rate of periprocedural stroke in the SAVR was less than $5.5 \%$ and greater than $6.0 \%$ in the TAVI arm (base-case complication rates shown in Table E1). The results of the probabilistic analysis indicated that approximately $52.7 \%$ and $55.4 \%$ of the 10,000 iterations fell below the $\$ 50,000$ and $\$ 100,000$ per QALY gained WTP threshold when TAVI was compared with SAVR, respectively. The costeffectiveness acceptability curve for TAVI versus SAVR, with a WTP threshold of $\$ 0$ to $\$ 200,000$, is shown in Figure 2.

In the transfemoral access scenario analysis, the index hospitalization cost was $\$ 40,210$ in the transfemoral TAVI arm and $\$ 29,632$ in the SAVR arm, and lifetime costs were $\$ 46,129 \pm \$ 4161$ and $\$ 36,314 \pm 7435$, respectively. The QALYs gained was $5.84 \pm 1.53$ and $5.44 \pm 1.48$ in TAVI and SAVR, respectively, yielding a difference of 0.40 gained for TAVI. The ICER, when restricted to the comparison of transfemoral TAVI to SAVR, was $\$ 24,790$ / QALY.

\section{CE Acceptability Curve}

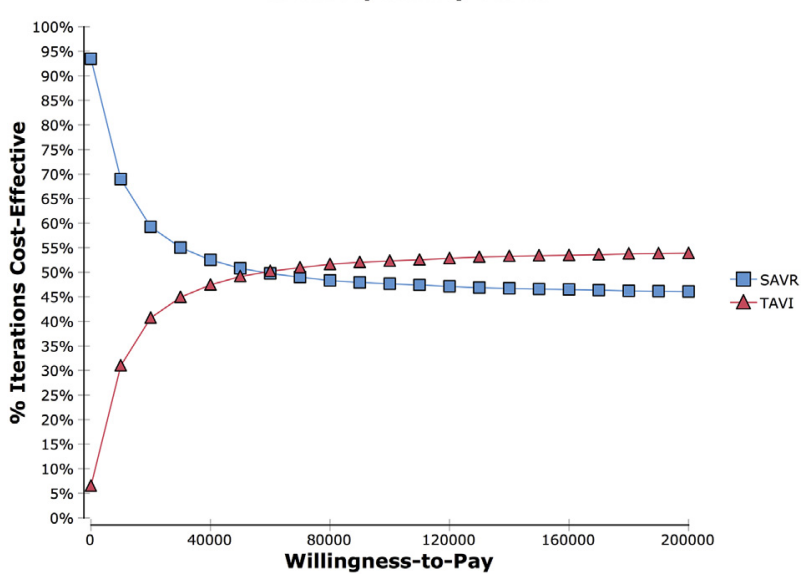

FIGURE 2. Cost-effectiveness acceptability curve for TAVI versus SAVR. The percent iterations of ICERs from the probabilistic analysis over the range of WTP from $\$ 0$ to $\$ 200,000$ are shown. $C E$, Cost-effectiveness; SAVR, surgical aortic valve replacement; TAVI, transcatheter aortic valve implantation.

\section{DISCUSSION}

In this cost-utility analysis based on contemporary evidence, we have shown that TAVI may be cost-effective in comparison with SAVR in the intermediate surgical risk population with a favorable ICER of \$46,083/QALY gained. This builds on numerous previous studies that have established that TAVI is an economically attractive option in the high surgical risk or the inoperable population. ${ }^{3}$ However, there was moderate-to-high uncertainty in our analyses, with the cost-effectiveness particularly sensitive to the cost of the TAVI prosthesis and the length of ICU stay.

TAVI is a Class IIa recommendation for the intermediaterisk population with severe AS in the most recent American Heart Association guidelines (2017). ${ }^{23}$ The intermediate surgical risk population represents a different population compared with the inoperable and high surgical risk population because these patients have substantially less comorbidities. $^{1,2,4}$ As such, their derived benefit from TAVI and associated costs differ. There is only 1 other study that compared costs in the intermediate-risk population using propensity score matching of 42 pairs of patients at a single Dutch institution. ${ }^{24}$ In this study, Osnabrugge and colleagues $^{24}$ found that initial in-hospital costs were higher in TAVI compared with the SAVR population (€40,802 vs $€ 33,354 ; P=.010$ ) and higher at 1 -year follow-up (total cost: $€ 46,217$ vs $€ 35,511 ; P=.009)$. The study did not consider effectiveness, but simply compared the costs of the interventions in a small sample of patients from a single institution over a 1-year time horizon. In contrast, our study used the recently published PARTNER 2 multicenter RCT data with more than 2000 patients to determine costs and effectiveness in a Markov model over a lifetime time horizon. Similar to the study by Osnabrugge and colleagues, ${ }^{24}$ our overall in-hospital costs at 30 days and lifetime follow-up costs were higher in the TAVI group compared with the SAVR group. Although costs were higher in the TAVI groups, the patients also accrued more QALYs over the lifetime horizon compared with SAVR in the basecase analysis. The cost findings of this study were similar to those reported by Wijeysundera and colleagues, ${ }^{25}$ who used micro-costing data from Ontario hospitals (TAVI, $\$ 42,742$; interquartile range, $\$ 37,295-\$ 56,196$ vs SAVR, $\$ 21,811$; interquartile range, $\$ 18,148-\$ 30,498)$. $^{25}$ 
Consistent with previous studies, ${ }^{8,25}$ our study found that the major cost-drivers were the cost of the TAVI prosthesis (TAVI vs SAVR: $\$ 24,000$ vs $\sim \$ 6000$ CAD), rates of complications, and length of stay in the ICU in our sensitivity analysis. Of note, TAVI was no longer cost-effective when the TAVI valve system cost was increased by $\$ 1100$ or when ICU LOS increased by 0.3 days. This highlights the impact of parameter uncertainty on the base-case results and the importance of conducting a fully probabilistic analysis such that all point estimates are drawn from specific distributions to address uncertainty. Nonetheless, our findings suggest that cost-savings through reduced ICU and hospital LOS help offset the higher acquisition cost of the TAVI valve system. In addition, the sensitivity of our analyses to these parameters reinforces the need for early discharge algorithms for patients undergoing TAVI to optimize length of stay, as well as increased market competition to reduce acquisitions costs for the prostheses themselves. ${ }^{26}$

The American College of Cardiology and American Heart Association consider a WTP threshold of greater than 3 times a country's gross domestic product (GDP) to be of low value, whereas a threshold between 1 and 3 times GDP is of intermediate value and less than a country's GDP to be high value. ${ }^{27}$ Thus, in both the Canadian and American perspective, ICERS less than \$50,000/QALY represent high value and ICERs between $\$ 50,000$ and $\$ 150,000$ / QALY represent intermediate value. Although the basecase analysis of our study would suggest that TAVI is a cost-effective alternative compared with SAVR in the intermediate-risk population, our probabilistic sensitivity analysis showed that TAVI was the preferred option in only $52.6 \%$ of simulations when the WTP threshold was $\$ 50,000 / \mathrm{QALY}$ gained (Figure 2). There was variation in terms of effectiveness and the ICER was inferior to SAVR in $41.7 \%$ of cases, representing moderate-to-high uncertainty around efficacy point estimates and the noninferiority nature of the results. Moreover, as highlighted earlier, even relatively small changes in the cost of the TAVI prosthesis or length of stay will have substantial impact on the overall cost-effectiveness. This underscores the limited evidence base in the intermediate-risk population and reinforces the need for additional clinical trials in this area.

Consistent with findings in the literature, the ICER in our scenario analysis of a transfemoral only cohort was lower than that of the entire cohort (transfemoral and transapical access) when compared with SAVR. ${ }^{28}$ The literature has shown lower index procedural and hospitalization costs along with improved outcomes in the transfemoral group compared with the transapical cohort. ${ }^{25}$ This difference likely is reflective of the older and more comorbid nature of the transapical cohort along with the less-invasiveness nature of transfemoral TAVI. Nonetheless, this reaffirms that transfemoral TAVI remains the preferred choice for access when possible. However, these results should be interpreted in the context of the design of the PARTNER 2 trial: The primary outcome was for the entire cohort, and secondary analyses considered stratifying by route of access.

The FDA has approved TAVI in the intermediate surgical risk population based on findings from the PARTNER 2 Trial. The intermediate-risk population already represents a substantial proportion of patients with severe AS treated with TAVI, accounting for $40 \%$ of patients in an analysis of the STS Transcatheter Valve Therapy database. ${ }^{29}$ Moreover, ongoing RCTs (NCT02825134, NCT02675114) are being conducted in the low-risk population (STS $<4 \%$ ). Multiple European registries have shown that TAVI has comparable results to SAVR in this even lower-risk population, ${ }^{30,31}$ representing another emerging group that may be eligible for TAVI in the near future. As the demand for TAVI increases, there are important implications for adapting existing infrastructure for care delivery. For example, accommodating increased TAVI use may require a shift in hospital priorities as greater capacity is needed for hybrid operating rooms or catheterization laboratories. A shift in human resources also may be needed as staff require additional training to perform these specialized procedures. These additional resources are typically not incorporated into a cost-utility analysis, but nonetheless are critical considerations.

\section{Study Limitations}

The interpretation of the results of our cost-utility analysis must be taken in the context of some limitations that merit discussion. Derivation of the initial procedural costs for SAVR and TAVI was obtained from expert opinion and thus may not be generalizable to other jurisdictions. Regardless, the rigor in costing data in the current study is confirmed because reported procedural costs were similar to previously published work from Ontario using microcosting data. ${ }^{25}$ Nonetheless, we acknowledge that the cost of medical care in the United States is substantially higher than that of the Canadian health care system. Although the costs may be higher in the US setting, they will likely be higher in both the TAVI and SAVR arms, so the incremental cost, and therefore ICER, would remain similar. Point estimates may vary slightly because of different cost inputs, but we do not expect a directional change. Furthermore, there was uncertainty around utility inputs in our study because the PARTNER 2 trial has yet to publish their utility study. However, the data we used from the PARTNER 1A trial of high-risk patients followed a similar pattern observed in other studies published in the literature, with patients in the TAVI group showing an earlier and more rapid improvement in QoL within the first 3 months of the procedure. ${ }^{21,32} \mathrm{~A}$ major limitation of our model is that the TAVI valve durability remains uncertain in the intermediate-risk population because the study only 
followed patients to 2 years. Currently, the best evidence to support valve durability is derived from the PARTNER 1A trial; there were no cases of structural valve deterioration at 5 years. ${ }^{33}$ Although patients in PARTNER 1A were older (mean age, 83.6-84.5 years) than patients in PARTNER 2 (mean age, 81.5-81.7 years), the average life years gained in our model was 7 years. ${ }^{2,4}$ Thus, we thought that valve durability would be similar to surgery over the lifetime of these octogenarians. However, extended follow-up of these patients will be of critical importance for low-risk young patients as TAVI indications evolve. Furthermore, findings from this study are generalizable to the intermediate-risk population only. The low-risk population may present with their own efficacy rates, complication rates, and length of stay that may affect the overall cost-effectiveness of TAVI. Last, PARTNER II only provided outcomes to 2 years; thus, after 2 years, we estimated the rate of mortality from Canadian life tables and assumed that these patients had a similar life expectancy to Canadians of similar age and gender. This methodology is consistent with previously published cost-effectiveness analyses. Furthermore, work by Mihaljevic and colleagues ${ }^{34}$ showed that life expectancy is restored to that of age- and gendermatched controls in patients aged more than 65 years undergoing aortic valve replacement for AS.

\section{CONCLUSIONS}

From the perspective of the Canadian health care system, compared with SAVR, TAVI may be a cost-effective option for the treatment of severe AS in the intermediate surgical risk population with an ICER of \$46,083/QALY in the base-case analysis. However, there was moderate to high uncertainty in our parameter estimates; only $52.7 \%$ and $55.4 \%$ of the ICERS in our simulations were below the WTP thresholds of $\$ 50,000$ and $\$ 100,000 /$ QALY, respectively. This meant that $44.6 \%$ to $47.3 \%$ of cases were not cost-effective. As such, despite the potential patient preference for a less-invasive approach, before widespread expansion of TAVI indications to lower-risk patients, more rigorous clinical evidence is needed.

\section{Conflict of Interest Statement}

H.C.W. has received research funding from Edwards Lifesciences and Medtronic Inc. S.E.F. serves as a proctor for Medtronic Inc. All other authors have nothing to disclose with regard to commercial support.

\section{References}

1. Leon MB, Smith CR, Mack M, Miller DC, Moses JW, Svensson LG, et al. Transcatheter aortic-valve implantation for aortic stenosis in patients who cannot undergo surgery. N Engl J Med. 2010;363:1597-607.

2. Smith CR, Leon MB, Mack MJ, Miller DC, Moses JW, Svensson LG, et al. Transcatheter versus surgical aortic-valve replacement in high-risk patients. $N$ Engl J Med. 2011;364:2187-98.
3. Sud M, Tam DY, Wijeysundera HC. The economics of transcatheter valve interventions. Can J Cardiol. 2017:33:1091-8.

4. Leon MB, Smith CR, Mack MJ, Makkar RR, Svensson LG, Kodali SK, et al. Transcatheter or surgical aortic-valve replacement in intermediate-risk patients. N Engl J Med. 2016;374:1609-20.

5. Tam DY, Vo TX, Wijeysundera HC, Ko DT, Rocha RV, Friedrich J, et al. Transcatheter vs surgical aortic valve replacement for aortic stenosis in low-intermediate risk patients: a meta-analysis. Can J Cardiol. 2017;33:1171-9.

6. Canadian Agency for Drugs and Technologies. Guidelines for the Economic Evaluation of Health Technologies: Canada. 4th ed. Ottawa, Canada: CADTH; 2017.

7. Statistics Canada. Life Tables, Canada, Provinces and Territories 2010 to 2012. Available at: http://www.statcan.gc.ca/pub/84-537-x/84-537-x2016006-eng. htm. Accessed November 5, 2016.

8. Doble B, Blackhouse G, Goeree R, Xie F. Cost-effectiveness of the Edwards SAPIEN transcatheter heart valve compared with standard management and surgical aortic valve replacement in patients with severe symptomatic aortic stenosis: a Canadian perspective. J Thorac Cardiovasc Surg. 2013;146: 52-60.e53.

9. Foley RN, Gilbertson DT, Murray T, Collins AJ. Long interdialytic interval and mortality among patients receiving hemodialysis. N Engl J Med. 2011;365: 1099-107.

10. Brønnum-Hansen H, Davidsen M, Thorvaldsen P. Danish MONICA Study Group. Long-term survival and causes of death after stroke. Stroke. 2001;32:2131-6.

11. Ministry of Health Long Term Care. Schedule of Benefits. Toronto: Queen's Printer; 2015.

12. Canadian Institute for Health Information. CIHI Patient Cost Estimator Website. Available at: https://www.cihi.ca/en/spending-and-health-workforce/spending/ patient-cost-estimator. Accessed November 1, 2016.

13. Canadian Institute for Health Information. Patient Cost Estimator: Methodological Notes and Glossary. Ottawa: CIHI; 2016.

14. Wong B, Courtney M, Pauly RP, Jindal K, Klarenbach S. Cost analysis of incentre nocturnal compared with conventional hemodialysis. Can J Kidney Health Dis. 2014;1:14.

15. Ohinmaa A, Zheng Y, Jeerakathil T, Klarenbach S, Häkkinen U, Nguyen T, et al. Trends and regional variation in hospital mortality, length of stay and cost in hospital of ischemic stroke patients in Alberta accompanying the provincial reorganization of stroke care. J Stroke Cerebrovasc Dis. 2016;25: 2844-50.

16. Kaier K, Gutmann A, Baumbach H, Zur Mühlen von C, Hehn P, Vach W, et al Quality of life among elderly patients undergoing transcatheter or surgical aortic valve replacement- a model-based longitudinal data analysis. Health Qual Life Outcomes. 2016;14:109.

17. Lanitis T, Cotté FE, Gaudin AF, Kachaner I, Kongnakorn T, Durand-Zaleski I Stroke prevention in patients with atrial fibrillation in France: comparative cost-effectiveness of new oral anticoagulants (apixaban, dabigatran, and rivaroxaban), warfarin, and aspirin. J Med Econ. 2014;17:587-98.

18. Tengs TO, Lin TH. A meta-analysis of quality-of-life estimates for stroke. Pharmacoeconomics. 2003;21:191-200.

19. Lee AJ, Morgan CL, Conway P, Currie CJ. Characterisation and comparison of health-related quality of life for patients with renal failure. Curr Med Res Opin. $2005 ; 21: 1777-83$.

20. Ambrosy AP, Hernandez AF, Armstrong PW, Butler J, Dunning A, Ezekowitz JA, et al. The clinical course of health status and association with outcomes in patients hospitalized for heart failure: insights from ASCEND-HF. Eur J Heart Fail. 2016;18:306-13.

21. Reynolds MR, Magnuson EA, Wang K, Thourani VH, Williams M, Zajarias A, et al. Health-related quality of life after transcatheter or surgical aortic valve replacement in high-risk patients with severe aortic stenosis. J Am Coll Cardiol. 2012;60:548-58.

22. Saw J, Bennell MC, Singh SM, Wijeysundera HC. Cost-effectiveness of left atrial appendage closure for stroke prevention in atrial fibrillation patients with contraindications to anticoagulation. Can J Cardiol. 2016;32:1355.e9-1355.e14.

23. Nishimura RA, Otto CM, Bonow RO, Carabello BA, Erwin JP, Fleisher LA, et al. 2017 AHA/ACC Focused Update of the 2014 AHA/ACC Guideline for the Management of Patients With Valvular Heart Disease: A Report of the American College of Cardiology/American Heart Association Task Force on Clinical Practice Guidelines. Circulation. 2017;135:e1159-95.

24. Osnabrugge RLJ, Head SJ, Genders TSS, Van Mieghem NM, de Jaegere PPT, van der Boon RMA, et al. Costs of transcatheter versus surgical aortic valve replacement in intermediate-risk patients. Ann Thorac Surg. 2012;94:1954-60. 
25. Wijeysundera HC, Li L, Braga V, Pazhaniappan N, Pardhan AM, Lian D, et al. Drivers of healthcare costs associated with the episode of care for surgical aortic valve replacement versus transcatheter aortic valve implantation. Open Heart. 2016;3:e00468.

26. Lauck SB, Wood DA, Baumbusch J, Kwon J-Y, Stub D, Achtem L, et al. Vancouver transcatheter aortic valve replacement clinical pathway: minimalist approach, standardized care, and discharge criteria to reduce length of stay. Circ Cardiovasc Qual Outcomes. 2016;9:312-21.

27. Anderson JL, Heidenreich PA, Barnett PG, Creager MA, Fonarow GC, Gibbons RJ, et al. ACC/AHA statement on cost/value methodology in clinical practice guidelines and performance measures: a report of the American College of Cardiology/ American Heart Association Task Force on Performance Measures and Task Force on Practice Guidelines. Circulation. 2014;129:2329-45.

28. Reynolds MR, Magnuson EA, Lei Y, Wang K, Vilain K, Li H, et al. Cost-effectiveness of transcatheter aortic valve replacement compared with surgical aortic valve replacement in high-risk patients with severe aortic stenosis: results of the PARTNER (Placement of Aortic Transcatheter Valves) trial (Cohort A). J Am Coll Cardiol. 2012;60:2683-92.

29. Holmes DR, Nishimura RA, Grover FL, Brindis RG, Carroll JD, Edwards FH, et al. Annual outcomes with transcatheter valve therapy: from the STS/ACC TVT Registry. J Am Coll Cardiol. 2015;66:2813-23.

30. Rosato S, Santini F, Barbanti M, Biancari F, D'Errigo P, Onorati F, et al. Transcatheter aortic valve implantation compared with surgical aortic valve replacement in low-risk patients. Circ Cardiovasc Interv. 2016;9:e003326.

31. Frerker C, Bestehorn K, Schlüter M, Bestehorn M, Hamm CW, Möllmann H, et al. In-hospital mortality in propensity-score matched low-risk patients undergoing routine isolated surgical or transfemoral transcatheter aortic valve replacement in 2014 in Germany. Clin Res Cardiol. 2017;106:610-7.

32. Cohen DJ. Health status benefits of transcatheter vs surgical aortic valve replacement in patients with severe aortic stenosis at intermediate surgical risk. Presented at: TCT 20167; November 1, 2016; Washington, DC. Available at: https://www.acc.orgT/media/Clinical/PDF-Files/Approved-PDFs/2016/10/25/ 07/TCT16_Nov01/950amET\%20PARTNER \%20II\%20QUALITY \%20OF\% 20LIFE.pdf. Accessed February 6, 2018.

33. Mack MJ, Leon MB, Smith CR, Miller DC, Moses JW, Tuzcu EM. 5-year outcomes of transcatheter aortic valve replacement or surgical aortic valve replacement for high surgical risk patients with aortic stenosis (PARTNER 1): a randomised controlled trial. Lancet. 2015;385:2477-84.

34. Mihaljevic T, Nowicki ER, Rajeswaran J, Blackstone EH, Lagazzi L, Thomas J, et al. Survival after valve replacement for aortic stenosis: implications for decision making. J Thorac Cardiovasc Surg. 2008;135:1270-9.

35. Maeda K, Kuratani T, Torikai K, Ichibori Y, Nakatani K, Onishi T, et al. Early outcomes in Japanese dialysis patients treated with transcatheter aortic valve implantation. Circ J. 2015;79:2713-9.

36. Brennan JM, Edwards FH, Zhao Y, O'Brien SM, Douglas PS, Peterson ED, et al. Long-term survival after aortic valve replacement among high-risk elderly patients in the United States: insights from the Society of Thoracic Surgeons Adult Cardiac Surgery Database, 1991 to 2007. Circulation. 2012;126:1621-9.

Key Words: Cost-effectiveness, TAVR, aortic stenosis, health economics

\section{Discussion \\ Scientific Session I}

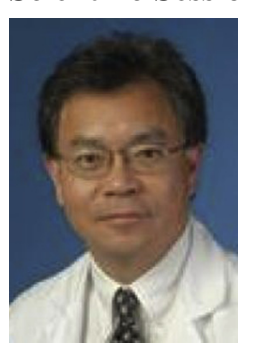

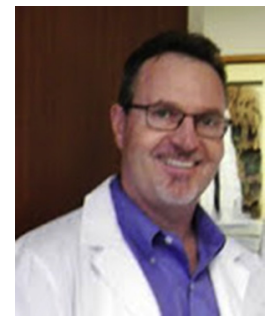

Moderator: R. M. Bremner

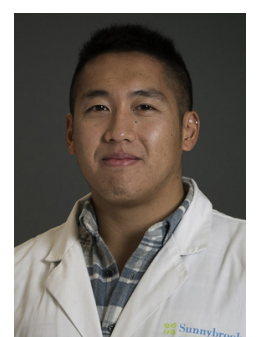

Dr Derrick Y. Tam. (Toronto, Ontario, Canada). On behalf of my collaborators, we thank you for the opportunity to present this work today. In addition, it is a privilege to have been selected as a finalist for the Paul C. Samson Resident Essay Prize. This morning, I'll be presenting a cost utility analysis of transcatheter versus SAVR for the treatment of AS in the intermediate surgical risk population. I have no disclosures.

TAVR has been shown to be cost-effective against medical therapy in the inoperable population and against SAVR or SAVR in the high surgical risk group. The recently published PARTNER 2 trials show noninferiority between TAVR and surgery at 30 days and 2 years. This was in the intermediate surgical risk population in whom the STS predicted risk of mortality was $4 \%$ to $8 \%$. On the basis of this evidence, the FDA has approved TAVR for the intermediate surgical risk population, and the most recent American Heart Association (AHA) valve guidelines have now made TAVR a class 2 recommendation for the intermediate-risk population. However, the cost-effectiveness has not been established in this group. As such, it is both relevant and timely to explore the cost-effectiveness of TAVR in the intermediate surgical risk group compared with surgery. Our population of interest is patients with severe AS at intermediate surgical risk. This is the PARTNER 2 cohort. Our intervention is the balloon-expandable TAVR performed transfemorally or transthoracically. Our comparator is surgery, and our outcomes of interest include lifetime effectiveness as measured by quality adjusted life years or "QALYs" and lifetime costs as measured in 2016 Canadian dollars. Our primary outcome is the ICER. This is calculated by taking the difference in lifetime costs between TAVR and surgery, also known as the incremental cost, divided by the difference in lifetime effectiveness between TAVR and surgery or the incremental effectiveness measured as QALYs. This gives us results in units of dollars per QALYs gained or QALYs gained. We used a fully probabilistic Markov model with 30-day cycle lengths, and we took the perspective of a third-party pair, which in our case is the Ontario Ministry of Health. We undertook a lifetime time horizon, and all costs and effectiveness were discounted at $1.5 \%$ per annum per the most recent guidelines. Our efficacy inputs came from the PARTNER 2 Trial, and our short-term cost 
inputs were mainly obtained from the Canadian Institute of Health Information, using their patient cost estimator, and from a variety of other sources. Our long-term costs and QoL inputs were obtained through a review of the literature. The following set of animations will describe the Markov model. We take a theoretical cohort of patients at intermediate surgical risk with severe AS. We model what happens to them when they undergo TAVR. We also model what happens to these patients when they undergo surgery. We then compare the 2 arms. Here we'll focus on the TAVR group, but the same thing happens with the surgical patients as well. All patients start off in the procedural state. Patients who undergo TAVR are at risk for a set of complications, including atrial fibrillation, acute kidney injury, stroke, rehospitalization, bleeding, and vascular injury. These are short-term states. They are also at risk for long-term complications, including dialysis, permanent stroke, and rehospitalization; they can also die as a result of the procedure or they can be discharged alive and well. With each of these complications, there are costs associated with it, and there are QoL utilities that are associated with it. The probabilities of being in each of these states are determined from the PARTNER 2 30-day outcomes. We run the same analysis for the surgical arm. After 30 days, the patients enter their long-term state. They enter their respective long-term state on the basis of the complications that took place in the first 30 days. They move along these different states, again from the probabilities from the PARTNER 2 1- and 2-year data. There are costs associated with each of these states, and there are utilities associated with each of these states or QoL metrics. We run the model until everyone is in the dead or the absorbing state, at which point the model stops, and we tabulate all costs and benefits for each arm and we compare the two.

In our base-case analysis, we used a fully probabilistic Markov model, which meant that all inputs were varied on the basis of its distribution in each iteration. The results shown are the average of 10,000 iterations or runs of the model. We also performed sensitivity analyses. One-way sensitivity analyses were performed, meaning that key parameters were varied one at a time to assess the impact on the ICER. We also looked at a transfemoral only cohort analysis using the subgroup analysis from the PARTNER 2 trial. These are the results of our base-case analysis.

Here we look at discounted costs and QALYs. We show that the total lifetime cost in the TAVR arm was $\$ 46,000$, whereas in the surgical arm it was $\$ 36,000$. Total lifetime QALYs gained were also higher in the TAVR group at 5.63, and it was 5.40 in the SAVR group. This meant that both costs and incremental effectiveness were higher in the TAVR arm. This yielded a final ICER of $\$ 46,000$ per QALY gained. To address the certainty, we used a probabilistic analysis. Any points that land in this quadrant would be more costly and effective. Any points in this quadrant would be more costly and less effective. Any points in this quadrant are less costly and less effective, and any points here would be less costly, but more effective. Each blue dot here represents 1 run of the model. There are 10,000 dots, 10,000 iterations of the model. If we take the average of those dots, we get our base-case analysis of $\$ 46,000$ per QALY. These 2 lines represent common willingness to pay thresholds by reimbursement agencies of $\$ 50,000$ and $\$ 100,000$ per QALY. Points to the right of those lines would be considered cost-effective. As you can see here, the cloud of blue dots are above the horizontal axis, meaning that on average, TAVR was more costly and there was a spread on the horizontal axis for incremental effectiveness, meaning that efficacy was evenly distributed between the 2 arms. We can also represent the previous graphic using a cost-effectiveness acceptability curve, and the red line here is the TAVR group. We show that they cost approximately $\$ 46,000$, which was our base-case ICER; approximately $50 \%$ of the iterations would be cost-effective at that point. As the willingness to pay increases, the number of iterations that are cost-effective tapers to approximately $56 \%$.

Here are the results of our 1-way sensitivity analysis. This is a tornado diagram. It shows that the cost of the TAVR valve has a great impact on the base-case ICER. It also shows that the ICU length of stay has an impact on our base-case ICER as well, along with 30-day mortality in both the surgical and TAVR arms. These are the results of our subgroup analysis of the transfemoral access only. We show again that the total lifetime costs are higher in the TAVR group but similar to the base-case analysis. However, the number of QALYs gained are higher in the TAVR group compared with the base-case analysis. This meant that the denominator is larger and as such yielded a lower ICER, approximately $\$ 24,000$ per QALYs. In our analysis, we showed that TAVR was cost-effective compared with surgery, at common willingness to pay thresholds. Compared with previous work that has shown cost-effectiveness in the inoperable population and the high-risk population, our ICER is slightly less than that of groups already being reimbursed. However, there was modest uncertainty surrounding the ICER; only 53\% of the ICERs fell below a $\$ 50,000$ threshold. I think this reflects a noninferiority nature of the data, and finally, the high acquisition costs of the TAVR valve may be offset by decreased ICU length of stay in the TAVR group and represents an area for TAVR improvement. However, the results of our study must be interpreted in the context of some limitations. The PARTNER-PARTNER 2 QoL data have not been published. As such, we had to use QoL data from previously published literature, the PARTNER-PARTNER 1A trial. There is a lack of perspective costing data from the 
PARTNER-PARTNER 2 trial; nonetheless, our reported costs are similar to published Ontario micro-costing data, and we believe that our methodology is rigorous.

Finally, contemporary postoperative practices have changed for TAVR, especially in 2017. There are early discharge algorithms, and in some centers, the ICU is bypassed entirely. This would reduce costs for the TAVR group.

In conclusion, we showed that TAVR is cost-effective, compared with surgery, at a willingness to pay a threshold of $\$ 50,000$ per QALY in the Canadian setting. There remain uncertainties surrounding the base-case ICER, and transfemoral access remains the preferred approach in patients undergoing TAVR. This was driven by improved effectiveness. Reduction in the cost of TAVR system and the reduced ICU length of stay will improve the cost-effectiveness of this technology in the future. Finally, I'd like to thank my supervisors, Dr Wijeysundera and Dr Fremes, and my collaborators.

Moderator. The discussion will be opened by $\mathrm{Dr}$ Michael Fischbein.

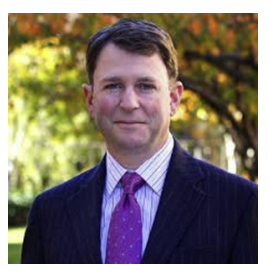

Dr Michael Fischbein. How do you account for valve durability in your cost-effective analysis? The population of the intermediate-risk group has been followed for only 2 years as you know, and there's unknown durability for TAVRs long-term in contrast to open aortic valve replacements. If patients require valve-on-valve in the future, especially in the younger population, how does this affect your analysis?

Dr Tam. That's a great point that you brought up with regard to valve durability, and you're right, in the PARTNER 2 trial, they only followed their patients to 2 years, so there's limited evidence to support valve durability in that study. To address your question with regard to reintervention, we didn't look at late reintervention in our model because it was actually small, the numbers, in the PARTNERPARTNER 2 trial. I think it was $1 \%$, and it was similar between both arms, so we didn't include that in our model. With respect to our assumptions with using valve durability data, I think the best evidence for this probably comes from the PARTNER 1A trial, and they have 5-year data to show that there wasn't really a difference in structural valve deterioration between the 2 arms. However, the patients in PARTNER 1 were older and had more comorbidities. In our model, our patients were 80 years old, and in our model, they lived for an additional 7 years when QoL was not accounted for. So, I think 7 and 5 years are similar, and it's a reasonable assumption that these patients probably won't have any structural valve deterioration within that timeframe. But again, you're right, it is a limitation of our study and requires further work, but I think this becomes more relevant when we look at expending indications for
TAVR to younger patients and low-risk patients, and I think this becomes critical to address then.

Dr Fischbein. For the second question, this cost analysis may be device specific. For example, will TAVR be as costeffective for the core valve where we know that the pacemaker rate is higher. Pacemakers will increase your numerator, the cost, and will also affect the quality, because these patients will be less satisfied as well.

Dr Tam. That's another excellent point, and this is actually something I'm really excited about because our group has just studied this and we've looked at a cost utility model for a self-expanding TAVR system using the SURTAVI data. We've recently submitted this to the STS as an abstract, so unfortunately I can't really describe the results for our findings for that study, but I do agree that you're right, this would affect the ICER, both the denominator and the numerator.

Dr Fischbein. The analysis is sensitive, as you mentioned, to changes in ICU length of stay, as well as in the step-down unit, and complications from the procedures, and so as you mentioned, in many institutions, patients are, especially the transfemorals, not going to the ICU. Instead, they're going directly to the step-down unit, and probably leaving the hospital in 2 or 3 days. But can these cost and practice patterns really be generalized to the ever-expanding number of TAVR programs that we're seeing even in the United States. I think there's more than 490 in 2016. So efficiency does come with practice, and as we see these smaller centers open, will they be able to discharge patients as quickly and have as low a complication rate, so will this cost analysis apply to everyone?

Dr Tam. Right. One of the limitations of any cost-effectiveness analysis is that it must be interpreted in a certain context and in certain jurisdictions, so it might not always apply. We use Canadian costs, but it's a single-payer system and a nonprofit system as well. But with respect to your question about efficiency, can smaller-volume hospitals discharge patients the same as large-volume hospitals? I think they can. I think in the United States, where you have a competitive marketplace and bundle payment schemes becoming more popular, this will force hospitals to become efficient when they do a procedure like TAVR. You also have health maintenance organizations that are good at organizing health care, and they might just combine centers that do TAVR or have TAVR centers of excellence once they realize that there are certain volume associations with outcomes.

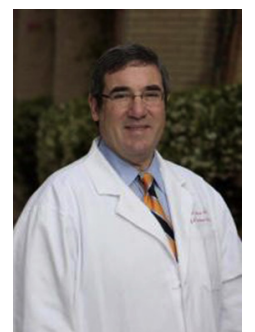

Dr R. Shemin (Los Angeles, Calif). Can you tell us whether you've compared your cost-effective analysis to other countries that have also done a similar study in their health care systems, and are there any differences or advantages that you'd like to say come from being able to do this type of study in Canada with a single-payer system? 
Dr Tam. That's an excellent question. This is actually the first cost-effectiveness analysis in the intermediate surgical risk population. This has been well studied in both the inoperable risk group, in which the risk of mortality is greater than $50 \%$ at 30 days, and the high surgical risk group across many jurisdictions, so in Europe, Canada, and even in the United States, this has been published. For most countries in the inoperable population, compared with medical therapy, it's very cost-effective, or even something we call dominant, where it's less costly but more effective. In the high surgical risk population, it does depend on jurisdiction as well. Is there a benefit to performing this in the Canadian setting? I think there is. The costs are fairly uniform in terms of how we source our costs and that adds rigor to our costing methodology, and we were able to show that using our costing methodology was similar to published Canadian data from previous groups.

Dr Shemin. To follow up on one of Dr Fischbein's questions, is there strong regionalization of TAVR sites in Canada? In the United States, we have an increasing number of programs that are doing what's called conscious sedation, where they're eliminating general anesthesia, patients are coming in the same day, limiting the ICU stay, which is one of your big drivers of cost, and how prevalent is that in your Canadian data, because clearly that's going to continue to advantage the TAVR population.

Dr Tam. Yes, that's an insightful comment and completely correct; as we remove the ICU from the equation, we know that the ICER will decrease. In our sensitivity analysis, we showed that if we just reduced the ICU length of stay in the TAVR group to 1 day, the ICER probably decreases to less than $\$ 40,000$ per QALY, so it's certainly affected. In Canada, there's approximately 27 centers that do TAVR. Our population is approximately 30 million, so one tenth of the United States. Most of these centers do less than 100 cases a year, and there are approximately 2 or 3 centers that do more than 100. A lot of them do use conscious sedation, and most of them are using step-down now instead of the ICU. So certainly, practices have changed since the publication of the PARTNER 2 trial.

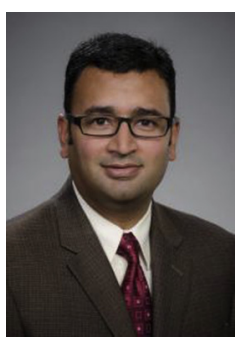

Dr N. Mokadam. You acknowledged in the article that because dialysis was not reported in the PARTNER 2 trial, you used PARTNER 1 assumptions. Those are different patients, and do you think that assumption is valid?

Dr Tam. Yes, that's a good point, and we struggled a bit with that when we were developing this model, but as with any economic model, there are limitations, especially when it's not built into a clinical trial. Sometimes we use what's called a patchwork to come up with a good model, and we use what we would consider the most similar group of patients to our model patients. I know PARTNER 1 patients are at slightly higher risk and they're slightly older, they're about 83 years old compared with 80 years. But if you have acute kidney injury, and you end up going to dialysis, I think the QoL outcomes are similar for that group. It is a smaller number of patients, though. 
TABLE E1. Base-case analysis efficacy inputs

\begin{tabular}{|c|c|c|c|c|c|c|c|}
\hline \multirow[b]{2}{*}{ Variable } & \multicolumn{3}{|c|}{ TAVI } & \multicolumn{3}{|c|}{ SAVR } & \multirow[b]{2}{*}{ Source } \\
\hline & $\overline{\text { Proportion }}$ & Alpha & Beta & Proportion & Alpha & Beta & \\
\hline \multicolumn{8}{|l|}{ Perioperative events $(<30 \mathrm{~d})$} \\
\hline Death & 0.039 & 39 & 972 & 0.040 & 41 & 980 & Leon and colleagues $(2016)^{4}$ \\
\hline Any stroke & 0.054 & 55 & 956 & 0.060 & 61 & 960 & Leon and colleagues $(2016)^{4}$ \\
\hline Disabling stroke* & 0.582 & 32 & 23 & 0.705 & 43 & 18 & Leon and colleagues (2016) \\
\hline Any renal failure & 0.013 & 13 & 998 & 0.030 & 31 & 990 & Leon and colleagues $(2016)^{4}$ \\
\hline Dialysis $\dagger$ & 0.714 & 10 & 4 & 0.714 & 10 & 4 & Smith and colleagues $(2011)^{2}$ \\
\hline Vascular injury & 0.079 & 80 & 931 & 0.050 & 51 & 970 & Leon and colleagues $(2016)^{4}$ \\
\hline Major bleeding & 0.104 & 105 & 906 & 0.433 & 442 & 579 & Leon and colleagues (2016) \\
\hline Atrial fibrillation & 0.090 & 91 & 920 & 0.260 & 265 & 756 & Leon and colleagues $(2016)^{4}$ \\
\hline Hospitalization & 0.064 & 64 & 947 & 0.061 & 62 & 959 & Leon and colleagues $(2016)^{4}$ \\
\hline \multicolumn{8}{|l|}{ Long-term events (year 1) } \\
\hline Death & 0.086 & 84 & 888 & 0.085 & 83 & 897 & Leon and colleagues (2016) \\
\hline Any stroke & 0.024 & 23 & 933 & 0.019 & 18 & 942 & Leon and colleagues (2016) \\
\hline Disabling stroke* & 0.708 & 17 & 7 & 0.684 & 13 & 6 & Leon and colleagues (2016) \\
\hline Any renal failure & 0.019 & 19 & 979 & 0.017 & 17 & 973 & Leon and colleagues $(2016)^{4}$ \\
\hline Dialysis & 0.714 & 10 & 4 & 0.714 & 10 & 4 & Smith and colleagues $(2011)^{2}$ \\
\hline Hospitalization & 0.082 & 78 & 869 & 0.076 & 73 & 886 & Leon and colleagues $(2016)^{4}$ \\
\hline Death on dialysis & 0.535 & 68 & 59 & 0.535 & 68 & 59 & Brennan and colleagues (2012) \\
\hline Any stroke on dialysis & 0.071 & 2277 & 29,788 & 0.071 & 2277 & 29,788 & Foley and colleagues $(2011)^{9}$ \\
\hline Disabling stroke & 0.077 & 1 & 12 & 0.077 & 1 & 12 & Maeda and colleagues (2015) \\
\hline Death related to stroke & 0.410 & 1701 & 2448 & 0.410 & 1701 & 2448 & Brønnum-Hansen and colleagues $(2001)^{10}$ \\
\hline Rehospitalization & 0.125 & 1525 & 10,657 & 0.125 & 1525 & 10,657 & Holmes and colleagues $(2015)^{29}$ \\
\hline \multicolumn{8}{|l|}{ Long-term events (year 2) } \\
\hline Death & 0.048 & 43 & 845 & 0.051 & 46 & 851 & Leon and colleagues $(2016)^{4}$ \\
\hline Any stroke & 0.014 & 13 & 920 & 0.006 & 6 & 936 & Leon and colleagues $(2016)^{4}$ \\
\hline Disabling stroke* & 0.769 & 10 & 3 & 0.625 & 5 & 3 & Leon and colleagues $(2016)^{4}$ \\
\hline Any renal failure & 0.004 & 4 & 975 & 0.009 & 9 & 964 & Leon and colleagues $(2016)^{4}$ \\
\hline Dialysis $\dagger$ & 0.714 & 10 & 4 & 0.714 & 10 & 4 & Smith and colleagues $(2011)^{2}$ \\
\hline Hospitalization & 0.047 & 41 & 828 & 0.024 & 21 & 865 & Leon and colleagues $(2016)^{4}$ \\
\hline Death on dialysis & 0.535 & 68 & 59 & 0.535 & 68 & 59 & Brennan and colleagues $(2012)^{36}$ \\
\hline Any stroke on dialysis & 0.071 & 2277 & 29,788 & 0.071 & 2277 & 29,788 & Foley and colleagues $(2011)^{9}$ \\
\hline Disabling stroke & 0.077 & 1 & 12 & 0.077 & 1 & 12 & Maeda and colleagues $(2015)^{35}$ \\
\hline Hospitalization during dialysis & 0.458 & 14,685 & 17,380 & 0.458 & 14,685 & 17,380 & Foley and colleagues $(2011)^{9}$ \\
\hline Death related to stroke & 0.410 & 1701 & 2448 & 0.410 & 1701 & 2448 & Brønnum-Hansen and colleagues $(2001)^{10}$ \\
\hline Rehospitalization & 0.125 & 1525 & 10,657 & 0.125 & 1525 & 10,657 & Holmes and colleagues $(2015)^{29}$ \\
\hline
\end{tabular}

Alpha and beta shape parameters were derived from event rates in the TAVI and SAVR arms as previously described by Doble and colleagues (2013). TAVI, Transcatheter aortic valve implantation; $S A V R$, surgical aortic valve replacement. *The rate of patients of disabling stroke in patients with any stroke. $\dagger$ Rate of patients with acute kidney injury who required subsequent dialysis postprocedure obtained from PARTNER 1A. 